\title{
Refractive Index Effects in Pendant Drop Tensiometry
}

\author{
Ziqing $\operatorname{Pan}^{1}$. J. P. Martin Trusler ${ }^{1}$ D
}

Received: 14 January 2022 / Accepted: 16 February 2022 / Published online: 26 February 2022

(c) The Author(s) 2022

\begin{abstract}
An optical model is established to investigate the effects of refractive index changes on the measurement of interfacial tension by the pendant drop method with axisymmetric drop shape analysis. In such measurements, light passes from the pendant drop through a surrounding bulk phase, an optical window and air to reach the lens of the camera system. The relation between object and image size is typically determined by calibration and, if the refractive indices of any of the materials in the optical path change between calibration and measurement, a correction should be made. The simple model derived in this paper allows corrections to be calculated along with the corresponding contribution to the overall uncertainty of the interfacial tension. The model was verified by measurements of the interfacial tension between decane and water under two different calibration conditions. Neglect of the correction was shown to cause errors of up to $6 \%$ when the bulk phase changed from air (during calibration) to water (during measurements) and of about $9 \%$ when the system was calibrated without the optical window used for the final measurements. The refraction changes due to high pressures and supercritical fluid states can also lead to measurement errors. The proposed model can facilitate more accurate interfacial tension measurements and reduce the amount of repetitive calibration work required.
\end{abstract}

Keywords Axisymmetric drop shape analysis - Calibration · Interfacial tension · Refractive index

\section{Introduction}

The pendant drop method is one of the most widely used techniques for determining surface tension and interfacial tension (IFT) [1-5]. It has the advantages of great accuracy and flexibility and is capable of measuring both static and dynamic IFTs over a wide range of values under either ambient conditions or high temperature,

J. P. Martin Trusler

m.trusler@imperial.ac.uk

1 Department of Chemical Engineering, Imperial College London, South Kensington Campus,

London SW7 2AZ, UK 
high pressure conditions [6-11]. Axisymmetric drop shape analysis (ADSA) is a powerful method for determining IFT based on the drop or bubble shape. The general procedure of ADSA is detection of the drop profile and numerical adjustment of the IFT such that the theoretical shape generated by the Young-Laplace equation matches the experimental drop profile $[12,13]$.

The surface or interfacial tension $\gamma$ is obtained from the relation

$$
\gamma=\Delta \rho g b^{2} / \beta,
$$

where $\Delta \rho$ is the difference between the densities of the two phases, $g$ is the gravitational acceleration, $b$ is the radius of curvature at the apex of the drop, and $\beta$ is the Bond number. Both $b$ and $\beta$ are determined through ASDA.

Prior to the measurement, a calibration is needed to determine the relation between the object and image size in the optical system, expressed practically as, e.g. pixels per mm. This is accomplished by imaging an object of known size [13-15]. Equation (1) indicates that the IFT is in a quadratic relation with the pixel size and so that relative errors in the pixel size are doubled in the measured surface or interfacial tension $[14,16]$. Refractive index changes in the optical path are one of the common causes of calibration error.

One approach is to include a suitable calibration standard within the interfacial tension view cell at the same distance from the camera as the drop to be imaged. Then an in situ calibration can be performed with the exact same optical path as used in the subsequent IFT measurement. The capillary used to create the pendant drop can serve this purpose but it is typically considerably smaller in diameter than the pendant drop, leading to high relative uncertainty, and it only serves to determine the calibration in one direction [17-19], leaving an uncertainty in the aspect ratio. Song and Springer [20] found that errors in the aspect ratio can have a large effect on the measured IFT. For example, a $1 \%$ error in the aspect ratio resulted in a $5 \%$ error in the IFT when the Bond number was 0.45. Moreover, repetitive calibration processes at each thermodynamic state point are tedious and time-consuming.

In practice, many researchers carry out calibrations with a sphere of precisely known diameter (e.g. $4 \mathrm{~mm}$ ), which has the advantage of determining the pixel size in two dimensions and hence also the aspect ratio [9, 21, 22]. Typically, this is done by placing the calibration sphere in the cell filled with ambient air, even when a different bulk phases will be present during the subsequent measurements. Closed interfacial tension cells use optical windows to allow the drop to be imaged and if these are replaced between calibration and measurement then there is another change in the optical path affecting the effective pixel size. In this paper, we present an elementary optical analysis that can account for these changes accurately leading to a simplified and less time-consuming calibration strategy. The same analysis can be used to estimate the contribution of uncertainties in the refractive indices of material in the optical path to the overall uncertainty of the IFT measurement.

Apart from pendant drop tensiometry, the present analysis may find application in other measurement techniques relying on optical imaging. For example, measurements of the viscosity and surface tension of liquid metals by the oscillating drop technique using electromagnetic or acoustic levitation or a microgravity 
environment $[23,24]$ may be affected by refractive index changes. Measurements of the density and thermal expansion coefficient of refractory materials in molten and solid phases via video-image processing of a levitated sample [25, 26] could be similarly influenced.

\section{Optical Model}

In the pendant drop method, a drop of the more-dense fluid is created at the end of a vertical capillary in a thermostatic view cell filled with the less-dense phase.

Alternatively, an inverse pendant drop of the less-dense fluid is created at the end of an inverted vertical capillary while the view cell is filled with the more-dense phase. The analysis of the two approaches is essentially the same. A source light is placed to one end of the view cell to illuminate the drop from behind and a CCD camera is placed at the other end. An image of the drop is captured by the CCD camera and analyzed by ADSA to determine the IFT between the two fluids according to Eq. (1). In this work, we developed an optical model to describe this process and investigated the effect of the refraction properties on the IFT measurement results.

Figure 1a shows the geometry of a typical interfacial tension setup for pendant drop tensiometry. AB is the object of interest, either the calibration tool or the pendant drop, with height $h_{0}$. The blue rectangle represents the bulk phase in the view cell, with $x_{\mathrm{c}}$ being the half-length of the view cell. The grey bars on either side of the object represent the windows with thickness $x_{\mathrm{w}}$. Light from the object passes sequentially through the bulk phase, the window and the air before reaching the lens, of focal length $f$, which generates a sharp image on the backplane of the CCD.

The detailed light path is shown in Fig. 1b. Light from point $\mathrm{A}$ is refracted at the bulk phase-window interface with angle of incidence $\theta_{1}$ and angle of refraction $\theta_{2}$. The light is refracted again at the window-air interface, with angle of incidence $\theta_{2}$ and angle of refraction $\theta_{3}$. Point $\mathrm{H}$ is the incidence point on the window-air interface. Line AE is parallel to the optical axis. The extension of line $\mathrm{OH}$ intersects line $\mathrm{AE}$ at point $\mathrm{A}^{\prime}$. It can be assumed that there is a hypothetic object $\mathrm{A}^{\prime} \mathrm{B}^{\prime}$, the image of which on the CCD backplane is CD.

According to Snell's law, the angles of incidence and refraction at the bulk phasewindow interface and the window-air interface have the following relationships:

$$
\begin{aligned}
& n_{\mathrm{b}} \sin \theta_{1}=n_{\mathrm{w}} \sin \theta_{2}, \\
& n_{\mathrm{w}} \sin \theta_{2}=n_{\mathrm{a}} \sin \theta_{3},
\end{aligned}
$$

where $n_{\mathrm{w}}, n_{\mathrm{a}}$ and $n_{\mathrm{b}}$ are the refractive indices of the window, air and the bulk phase inside the cell, respectively.

In a typical interfacial tension measurement setup, the size of the pendant drop or the calibration tool is of the order of millimeters, while length of the optical axis is of meter scale. Therefore, the angles of incidence and refraction are small and we can 


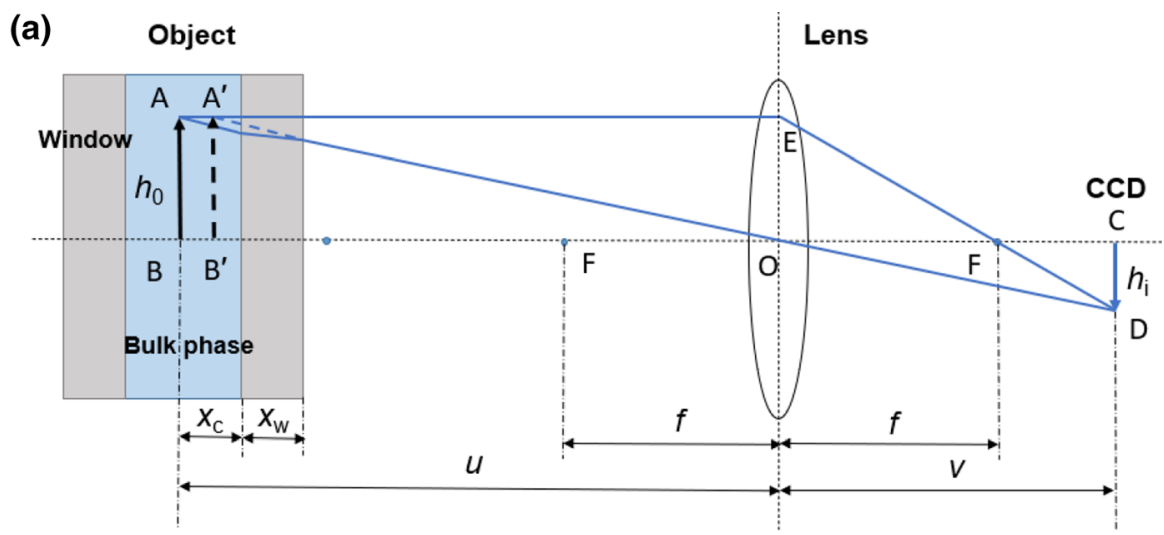

(b)

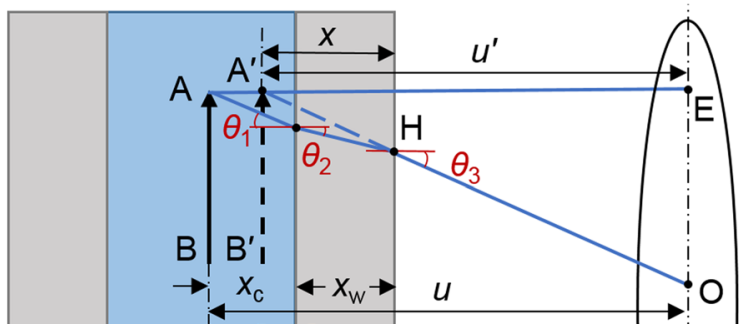

Fig. 1 (a) Geometrical description of a typical pendant drop setup for IFT measurements. (b) Detailed light path from the object through the bulk phase in the view cell, the window, air and the lens. Dimensions: $h_{0}$, height of the object; $x_{\mathrm{c}}$, half-length of the view cell; $x_{\mathrm{w}}$, thickness of the window; $u$, distance between the object and the lens; $f$, focal length of the lens; $v$, distance between the lens and the CCD backplane; $h_{\mathrm{i}}$, height of the image; $x$, distance between the hypothetic object and the window-air interface; $u^{\prime}$, distance between the hypothetical object and the lens; $\theta_{1}$, angle of incidence at the bulk phasewindow interface; $\theta_{2}$, the refraction angle at the bulk phase-window interface; $\theta_{3}$, the refraction angle at the window-air interface

approximate $\sin \theta_{i} \approx \tan \theta_{i}$, where $i=1,2,3$. The following geometric relationships can also be obtained to facilitate the solution of the model:

$$
x_{\mathrm{c}} \tan \theta_{1}+x_{\mathrm{w}} \tan \theta_{2}=x \tan \theta_{3}
$$

The distance between the hypothetic object $\mathrm{A}^{\prime} \mathrm{B}^{\prime}$ and the lens is

$$
u^{\prime}=u-x_{\mathrm{c}}-x_{\mathrm{w}}+x
$$

where $u^{\prime}$ is the hypothetic object distance and $u$ is the real object distance. According to the Gaussian lens formula, the distance between the lens and the image can be determined as follows:

$$
\frac{1}{f}=\frac{1}{u^{\prime}}+\frac{1}{v},
$$




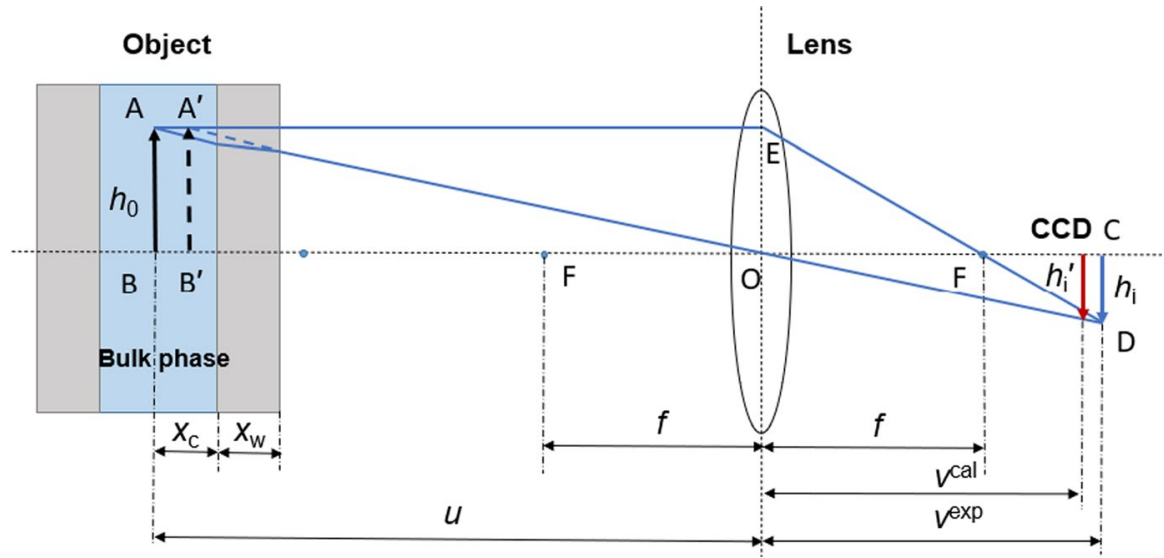

Fig. 2 Projection of the theoretical image on the CCD camera sensor

where $f$ is the focal length of the CCD camera and $v$ is the image distance. Consequently, the height of the image $h_{\mathrm{i}}$ can be determined with the geometric relationship:

$$
h_{\mathrm{i}}=h_{0} \cdot \frac{v}{u^{\prime}},
$$

where $h_{0}$ denotes the size of the object.

Prior to calibration, the primary lens is moved relative to the projection plane to obtain a sharp image. Once the calibration is completed, the lens should not be moved. However, if the refractive properties of any of the optical elements (the bulk phase in the view cell, the window or the lens) are changed, the focal plane will no longer fall on the CCD backplane. The size of the image created on the CCD backplane is then not the theoretical image size but the projection of the theoretical image onto the sensor, as shown in Fig. 2. The height $h_{\mathrm{i}}{ }^{\prime}$ of the real image captured by the camera is given by

$$
h_{\mathrm{i}}^{\prime}=h_{\mathrm{i}}\left(v^{\mathrm{cal}} / v^{\exp }\right)
$$

where $h_{\mathrm{i}}$ is the theoretical image height, $v^{\mathrm{cal}}$ is the image distance during the calibration, i.e. the distance between the lens and the CCD backplane, and $v^{\exp }$ is the theoretical image distance during the experiment.

The correct IFT $\gamma$ should be calculated with the effective pixel length under the experimental conditions, while the value $\gamma_{0}$ obtained using the pixel length under the calibration conditions requires correction. Equation (1) shows that the IFT is in a quadratic relationship with length and therefore the corrected IFT can be obtained from the relation

$$
\gamma=\gamma_{0}\left(l^{\exp } / l^{\mathrm{cal}}\right)^{2}
$$

where $l$ is pixel length and superscripts 'exp' and 'cal' denote values under experimental and calibration conditions, respectively. In the calibration, the pixel length is 
Table 1 Description of the materials used, where $w$ denotes mass fraction and $\rho_{e}$ denotes electrical resistivity

\begin{tabular}{lllll}
\hline Chemical name & CAS number & Source & Purity as supplied & Additional purification \\
\hline Deionized water & $7732-18-5$ & $\begin{array}{c}\text { Millipore } \\
\text { Direct-Q UV3 } \\
\text { apparatus }\end{array}$ & $\begin{array}{c}\rho_{e} \geq 18 \mathrm{M} \Omega \cdot \mathrm{cm} \text { at } \\
T=298 \mathrm{~K}\end{array}$ & Vacuum degassed \\
Decane & $124-18-5$ & $\begin{array}{c}\text { Sigma Aldrich } \\
\text { igmation by activated }\end{array}$ & $\begin{array}{c}\text { Adsorption } \\
\text { alumina }\end{array}$ \\
\hline
\end{tabular}

determined from the size of the calibration tool and the number of pixels its image occupies. For the same calibration tool, the calculated pixel length is inversely proportional to the number of pixels, i.e., inversely proportional to the size of the image. Therefore, the corrected IFT can be given by

$$
\gamma=\gamma_{0}\left(h_{\mathrm{i}}^{\text {cal }} / h_{\mathrm{i}}^{\text {exp }}\right)^{2}
$$

To sum up, the correction of the IFT due to refractive index change can be expressed as

$$
\gamma=\gamma^{\exp } \cdot\left[\frac{u+\left(n_{\mathrm{a}} / n_{\mathrm{b}}^{\exp }-1\right) x_{\mathrm{c}}+\left(n_{\mathrm{a}} / n_{\mathrm{w}}^{\exp }-1\right) x_{\mathrm{w}}}{u+\left(n_{\mathrm{a}} / n_{\mathrm{b}}^{\text {cal }}-1\right) x_{\mathrm{c}}+\left(n_{\mathrm{a}} / n_{\mathrm{w}}^{\text {cal }}-1\right) x_{\mathrm{w}}}\right]^{2} .
$$

If the window is removed during calibration, $n_{\mathrm{w}}$ air $=n_{\mathrm{a}}$.

\section{Experimental Section}

To verify the optical model, we calibrated the tensiometer with a stainless-steel sphere of known diameter in air, in water and in air without the viewing window. IFTs between DI water and decane were also measured with the calibration parameters obtained in air and the calibration parameters obtained in water.

\subsection{Materials}

Table 1 details the fluids studied.

Prior to the measurement, the deionized water was degassed under vacuum for $20 \mathrm{~min}$ and decane was passed through a $500 \mathrm{~mm}$ long chromatography column filled with activated alumina powder (100 mesh) to remove the surface-active impurities, which are believed to cause the IFT to drift downwards over time [27, 28].

\subsection{Methodology}

A well-established pendant drop tensiometer (Ramé-Hart Instrument Co.) was used to carry out the IFT measurement. The main component is a thermostatic view cell 
with sapphire windows fitted at each end. A diffuse LED light source (CREE) was employed to illuminate the pendant drop while a monochrome CCD camera (RaméHart Instrument Co.) was employed to capture the drop image. Further details of this apparatus can be found elsewhere [29]. The standard uncertainty of the temperature measurement is $0.025 \mathrm{~K}$ and the standard uncertainty of the pressure measurement is $0.035 \mathrm{MPa}$.

The calibration tool, a high-precision stainless-steel ball provided by RaméHart Instrument Co. was used to carry out the calibration of the tensiometer. The diameter of the sphere was $(4.000 \pm 0.001) \mathrm{mm}$. Calibration against a sphere was selected because it considers both the horizontal and the vertical directions. Calibrations were carried out under three conditions: (1) the view cell full of air and the viewing window removed; (2) the view cell full of air and the viewing windows installed; (3) the view cell full of water and the viewing window installed. The sapphire window closest to the CCD camera was first removed and the calibration tool was put inside the view cell at the place where the pendant drop is normally located. The light intensity and lens aperture were adjusted to obtain a sharp image with a well-defined profile. The software determined the equivalent pixel lengths $I_{z}$ in the vertical direction and $I_{x}$ in the horizontal direction for this first condition. The sapphire window that had been removed was then replaced and the pixel lengths $I_{z}$ and $I_{x}$ were recorded in this second condition. Finally, DI water was injected into the view cell until the calibration tool was fully submerged and the pixel lengths $I_{z}$ and $I_{x}$ were recorded in this third condition. All the three calibrations were carried out at ambient temperature of $(295.21 \pm 0.04) \mathrm{K}$.

To investigate the effect of the refractive index changes on IFT measurements, the IFT between decane and water was measured with the calibration parameters obtained both in water and in air with the window in place. The view cell was first filled with water. Decane was then slowly injected into the view cell through the capillary until a well-shaped inverse pendant drop was created at the tip of the capillary. The image of the pendant drop was captured by the camera every three seconds and analyzed by the image processing software Advanced DROPimage [Ramé-Hart Instrument Co.]. At least three drops were measured to evaluate the reproducibility of the measurement. The first measurement was implemented with the calibration parameters obtained in air and the second measurement was implemented with the calibration parameters obtained in water.

\section{Results and Discussions}

\subsection{Verification of the Model with the Calibration Results}

The calibration results obtained in each of the three configurations described above are reported in Table 2 and Fig. 3. The calibration in air without the viewing window in place had the largest pixel lengths, which were about $5.1 \%$ larger than the pixel lengths calibrated in air with both windows and about $7.6 \%$ larger than the pixel lengths calibrated in water. Since the image size is inversely proportional to the calibrated pixel length, the image of the calibration sphere in condition (2) is 
Table 2 Horizontal pixel length $l_{\mathrm{x}}$, vertical pixel length $l_{\mathrm{z}}$ and the aspect ratio under three calibration conditions: (1) in air with the window closest to the lens removed; (2) in air with both windows; (3) in water with both windows

\begin{tabular}{llll}
\hline Conditions & $l_{\mathrm{x}}(\mu \mathrm{m} / \mathrm{pixel})$ & $l_{\mathrm{z}}(\mu \mathrm{m} / \mathrm{pixel})$ & Aspect ratio \\
\hline 1 & 15.771 & 15.753 & 1.0012 \\
2 & 14.981 & 15.000 & 0.9987 \\
3 & 14.638 & 14.654 & 0.9989 \\
\hline
\end{tabular}
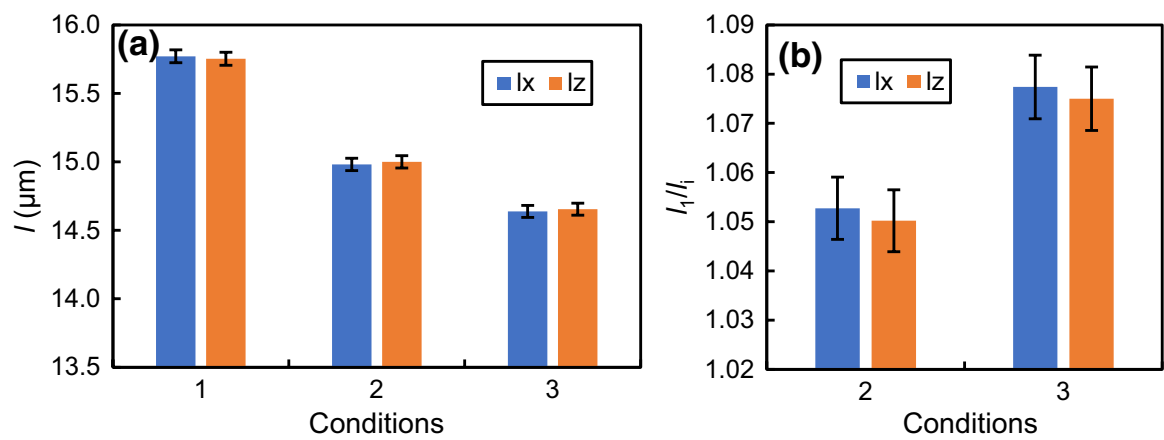

Fig. 3 (a) Horizontal pixel length $l_{\mathrm{x}}$ and vertical pixel length $l_{\mathrm{z}}$ under three calibration conditions: (1) in air without the window closest to the lens; (2) in air with both windows; (3) in water with both windows. (b) The ratio of the pixel length under condition (1) to the pixel length under condition $(i), i=2,3$

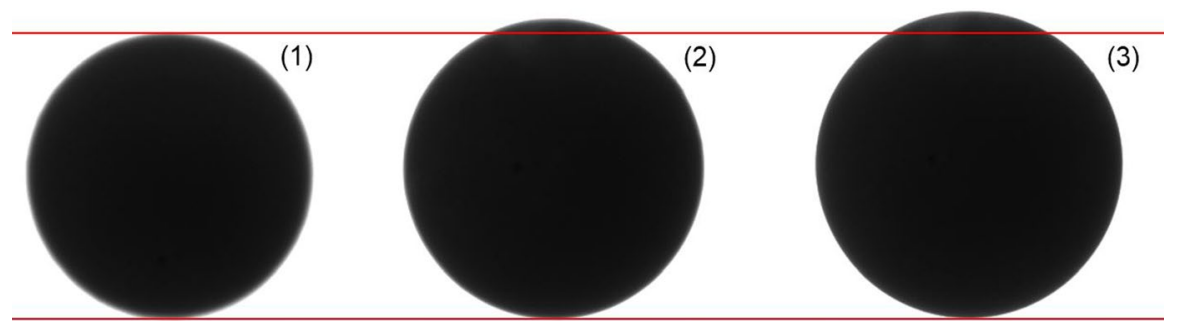

Fig. 4 Images of the calibration sphere under three calibration conditions: (1) in air without the window closest to the lens; (2) in air with both windows; (3) in water with both windows. Size $(1)<\operatorname{size}(2)<$ size (3)

about $5.1 \%$ larger than in condition (1), while that in condition (3) is about $7.6 \%$ larger than in condition (1). This is illustrated by the captured images of the calibration sphere under the three conditions as shown in Fig. 4.

The image size of the calibration sphere under different conditions can be predicted with the optical model proposed in Sect. 2. The basic parameters of the interfacial tension setup are listed in Table 3.

The refractive index of air under ambient conditions is 1.0003 [30], the refractive index of water under ambient conditions is 1.333 [31] and the refractive index 
Table 3 Basic parameters of the interfacial tension setup

\begin{tabular}{ll}
\hline Parameter & Value \\
\hline Object distance, $u(\mathrm{~mm})$ & 220 \\
Half-length of the view cell, $x_{\mathrm{c}}(\mathrm{mm})$ & 24 \\
Thickness of the sapphire window, $x_{\mathrm{w}}(\mathrm{mm})$ & 22 \\
Focal distance of the lens, $f(\mathrm{~mm})$ & 75 \\
Diameter of the calibration sphere $(\mathrm{mm})$ & 4.000 \\
\hline
\end{tabular}

Fig. 5 Comparison of the experimentally determined ratio and the calculated ratio of the pixel length under condition (1) to the pixel length under condition $i$, where $i=2,3$

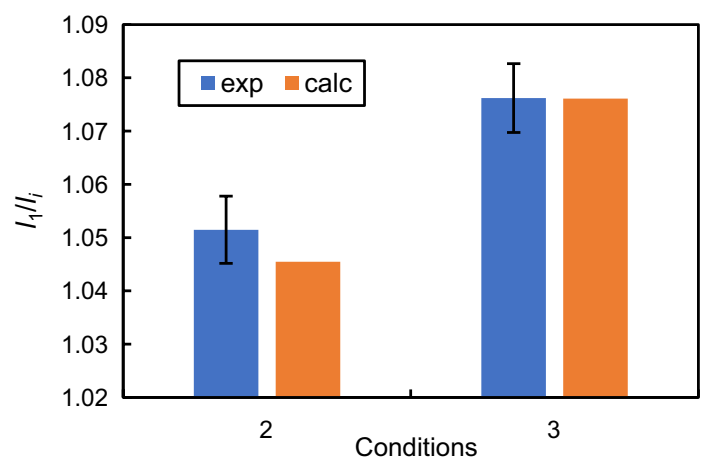

of sapphire under ambient condition is 1.77 [32]. The predicted image size of the calibration sphere under condition (1), (2) and (3) are $53.0 \%, 55.4 \%$ and $57.0 \%$ of the calibration sphere diameter, respectively. Figure 5 shows a comparison of the experimentally determined pixel size and the calculated pixel size in terms of the ratio of the pixel size under condition (1) to the pixel size under condition $i$, where $i=2,3$. It can be concluded that the modeling results are in good agreement with the experimental results, considering the uncertainty in the length measurement and the uncertainty in the drop edge detection.

\subsection{Verification of the Model with the Measured IFTs}

In order to verify the optical model experimentally in terms of IFT, the calibration of the tensiometer was first carried out in air. After that, the IFT between decane and water was measured at $T=294.15 \mathrm{~K}$ and $p=0.1 \mathrm{MPa}$. The tensiometer was then calibrated in water, and the IFT between decane and water was measured again under the same experimental conditions.

The IFTs obtained with the calibration parameters in air was further corrected according to Eq. (11). Figure 6 shows a comparison among the IFTs between decane and water under the same experimental conditions but different calibration conditions. Compared with the IFT data reported by other authors in Table 4, both calibration in water and calibration in air followed by correction with the optical model gave good results. 
Fig. 6 IFTs between decane and water at $T=294 \mathrm{~K}, p=0.1 \mathrm{MPa}$. $\bigcirc$, calibrated in air; $\square$, calibrated in air and corrected by Eq. (11); $\Delta$, calibrated in water

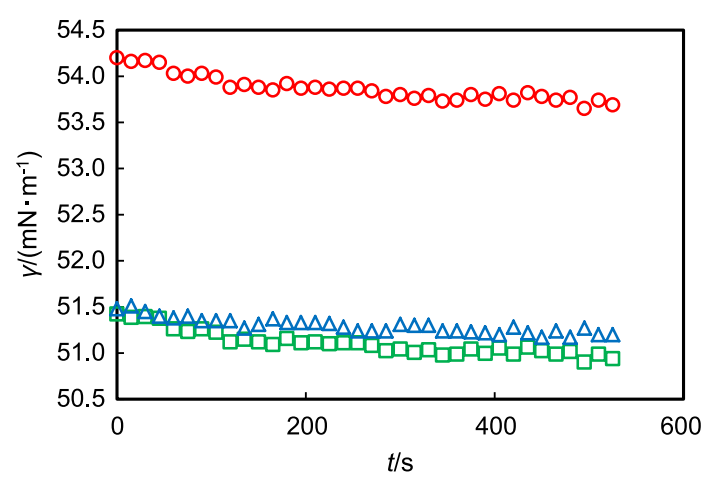

\subsection{Effect of the Refractive Index on the Measured IFTs}

We consider the basic parameters of the model presented in Sect. 4.1 to investigate the effect of refractive index on IFT measurements. In the following case studies, we assume that the calibration is carried out under ambient conditions when the bulk phase inside the view cell is air.

If the refractive index of the bulk phase in the view cell is different from that of the bulk phase during calibration, measurement error will occur. Figure 7 shows the relative error of the IFT as a function of the refractive index of the experimental bulk phase in the view cell.

Table 5 lists the refractive indices of some common materials and the relative error of the measured IFT if the calibration was carried out in air. There are substantial measurement errors when the bulk phase in the view cell is a liquid as the refractive indices of liquid and air are very different. Therefore, either the calibration condition has to be the same with the experimental condition or a correction has to be applied to the measured IFTs.

Another error would be to calibrate without the window but then make the measurements with it replaced. In the case of a sapphire window with the thickness of $22 \mathrm{~mm}$, the measured IFTs will be higher than the actual values with a relative error of $9.3 \%$.

In addition, different thermodynamic conditions between the calibration and the experimental states can lead to measurement error, especially for measurements under high pressure or supercritical states. Figure 8 shows the relative error of the

Table 4 IFT between decane and water reported in the literatures

\begin{tabular}{llll}
\hline Author & $T(\mathrm{~K})$ & $p(\mathrm{MPa})$ & $\gamma\left(\mathrm{mN} \bullet \mathrm{m}^{-1}\right)$ \\
\hline Kodera et al. [33] & 293.1 & 0.01 & 51.40 \\
Aveyard and Haydon [34] & 293.1 & 0.1 & 52.30 \\
Zeppieri et al. [28] & 293.1 & 0.1 & 52.33 \\
Wiegand et al. [35] & 295 & 0.1 & 51.1 \\
Sayed et al. [22] & 295 & 0.1 & 51.1 \\
Georgiadis et al. [5] & 297.85 & 0.1 & 51.96 \\
\hline
\end{tabular}


Fig. 7 Relative error of the IFT as a function of refractive index $n_{\text {media }}$ of the bulk phase, where $\gamma_{\mathrm{m}}$ denotes the measured IFT and $\gamma_{0}$ denotes the actual IFT

Table 5 Refractive indices of some common materials at $T=298.15 \mathrm{~K}$ and $p=0.1 \mathrm{MPa}$ and the relative error of the measured IFT when calibration was carried out in ambient air

Fig. 8 Relative error of the measured IFT with the bulk phase of $\mathrm{N}_{2}$ as a function of the pressure at a temperature of $323.15 \mathrm{~K}$, where $\gamma_{\mathrm{m}}$ denotes the measured IFT and $\gamma_{0}$ denotes the actual IFT

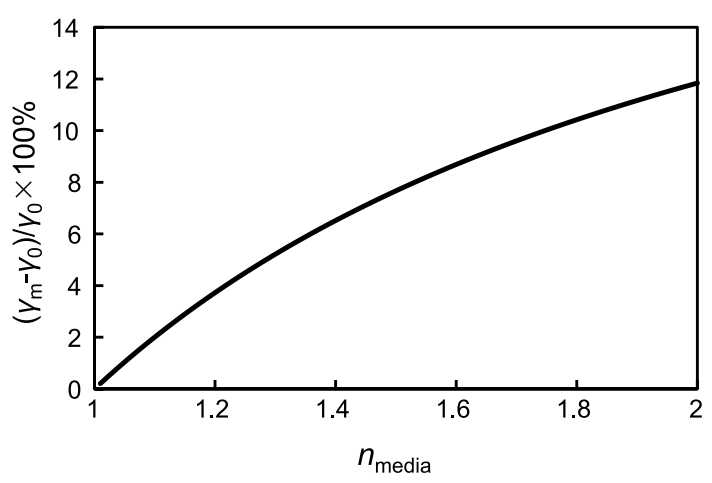

\begin{tabular}{llll}
\hline Materials & Refractive index & Reference & $\frac{\gamma_{\mathrm{m}}-\gamma_{0}}{\gamma_{0}}(\%)$ \\
\hline $\mathrm{CO}_{2}$ & 1.0005 & {$[36]$} & 0.003 \\
DI water & 1.3330 & {$[31]$} & 5.9 \\
Ethanol & 1.3610 & {$[37]$} & 6.3 \\
Acetone & 1.3590 & {$[38]$} & 6.3 \\
Benzene & 1.4986 & {$[39]$} & 8.0 \\
Decane & 1.4099 & {$[40]$} & 7.0 \\
R123a & 1.3291 & {$[41]$} & 5.9 \\
Virgin olive oil & 1.4676 & {$[42]$} & 7.7 \\
\hline
\end{tabular}

measured IFT with a bulk phase of $\mathrm{N}_{2}$ as a function of pressure when the calibration is conducted at ambient pressure. The refractive index data of $\mathrm{N}_{2}$ were taken from [43]. The results indicate that the measurement error increases with increasing experimental pressure. At $28.7 \mathrm{MPa}$, the measurement error is $1.4 \%$. Figure 9 shows a similar analysis for the case in which the bulk phase is $\mathrm{CO}_{2}$ at different temperatures such that gas, liquid and supercritical states occur. The refractive index data of $\mathrm{CO}_{2}$ were taken from [44]. The relative error increases with increasing pressure and decreases with increasing temperature, essentially reflecting the behavior 
of the density. For example, in the saturated liquid at a temperature of $298.15 \mathrm{~K}$, the relative error is $3.4 \%$.

From the preceding discussions, it can be concluded that either the calibration should be implemented under the same conditions and with the same bulk media as in the subsequent experiments or a correction should be applied. With the proposed optical model, corrections can be calculated accurately and tedious calibration work at each thermodynamic state point can be avoided. Only one calibration in air under ambient conditions is needed, while validation can be achieved in situ at any state point by imaging the capillary tip. The refractive index of the experimental bulk phase inside the view cell can either be obtained from the literature or be calculated from the Lorentz-Lorenz equation:

$$
\frac{n^{2}-1}{n^{2}+2}=\frac{4 \pi N_{\mathrm{A}} \alpha}{3 V_{\mathrm{m}}} .
$$

Here, $n$ is the refractive index of the substance, $N_{\mathrm{A}}$ is Avogadro's constant, $\alpha$ is the molecular polarizability, and $V_{\mathrm{m}}$ is the molar volume.

\section{Conclusions}

An optical model is proposed to quantify the effect of refractive index changes on the measurement of IFT by pendant drop tensiometry. The model was validated experimentally by determining the image pixel length under three different calibration conditions. The experimentally determined image size in air with both windows is $5.1 \%$ larger than that in air without a window between the camera and the object, while the image size obtained in water with both windows is $7.6 \%$ larger than that in air with the window closest to the camera removed. In comparison, the predicted changes are $4.6 \%$ and $7.6 \%$, respectively. The model was further verified by the measured IFT between decane and water. The IFT with the calibration parameters obtained in water and the calibration parameters obtained in air, corrected according to the model, are in good agreement with the reference data, while the IFT obtained with the calibration parameters in air without any correction deviate significantly from the reference data.

Fig. 9 Relative error of the measured IFT with the bulk phase of $\mathrm{CO}_{2}$ as a function of pressure at different temperatures, where $\gamma_{\mathrm{m}}$ denotes the measured IFT and $\gamma_{0}$ denotes the actual IFT

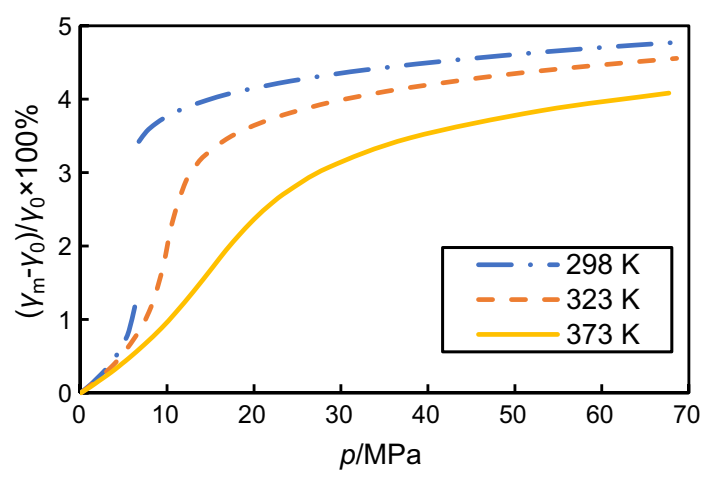


Changes in the refractive index of the bulk phase, for example as a result of high pressure or supercritical states, can also significantly influence result when the calibration is implemented under the ambient condition. Nevertheless, the proposed model can be applied to correct the measurement results reliably and avoid the need for repetitive and complex calibration work. The uncertainty of the bulk media refractive index can be used to estimate the uncertainty of the correction.

Funding The authors gratefully acknowledge the Imperial-CSC scholarship jointly funded by Imperial College London and China Scholarship Council (CSC).

Open Access This article is licensed under a Creative Commons Attribution 4.0 International License, which permits use, sharing, adaptation, distribution and reproduction in any medium or format, as long as you give appropriate credit to the original author(s) and the source, provide a link to the Creative Commons licence, and indicate if changes were made. The images or other third party material in this article are included in the article's Creative Commons licence, unless indicated otherwise in a credit line to the material. If material is not included in the article's Creative Commons licence and your intended use is not permitted by statutory regulation or exceeds the permitted use, you will need to obtain permission directly from the copyright holder. To view a copy of this licence, visit http://creativecommons.org/licen ses/by/4.0/.

\section{References}

1. C.E. Stauffer, J. Phys. Chem 69(6), 1933 (1965)

2. J.D. Berry, M.J. Neeson, R.R. Dagastine, D.Y.C. Chan, R.F. Tabor, J. Colloid Interface Sci. 454, 226 (2015)

3. F.K. Hansen, G. Rødsrud, J. Colloid Interface Sci. 141(1), 1 (1991)

4. M. Ghorbani, A.H. Mohammadi, J. Mol. Liq. 227, 318 (2017)

5. A. Georgiadis, G. Maitland, J.P.M. Trusler, A. Bismarck, J. Chem. Eng. Data 56(12), 4900 (2011)

6. Z. Yang, X. Liu, Z. Hua, Y. Ling, M. Li, M. Lin, Z. Dong, Colloids Surf. A Physicochem. Eng. Asp. 482, 611 (2015)

7. S. Al-Anssari, A. Barifcani, A. Keshavarz, S. Iglauer, J. Colloid Interface Sci. 532, 136 (2018)

8. Y.T.F. Chow, G.C. Maitland, J.P.M. Trusler, J. Chem. Thermodyn. 93, 392 (2016)

9. X. Li, E. Boek, G.C. Maitland, J.P.M. Trusler, J. Chem. Eng. Data 57(4), 1078 (2012)

10. Y. Touhami, D. Rana, V. Hornof, G.H. Neale, J. Colloid Interface Sci. 239(1), 226 (2001)

11. X. Wang, L. Liu, Z. Lun, C. Lv, R. Wang, H. Wang, D. Zhang, J. Spectrosc. (Hindawi) 2015, 285891 (2015)

12. O.I.D. Rio, A.W. Neumann, J. Colloid Interface Sci. 196(2), 136 (1997)

13. M. Hoorfar, A.W. Neumann, Adv. Colloid Interface Sci. 121(1), 25 (2006)

14. G. Loglio, P. Pandolfini, A.V. Makievski, R. Miller, J. Colloid Interface Sci. 265(1), 161 (2003)

15. P. Cheng, D. Li, L. Boruvka, Y. Rotenberg, A.W. Neumann, Colloids Surf. 43(2), 151 (1990)

16. Y. Touhami, G.H. Neale, V. Hornof, H. Khalfalah, Colloids Surf. A Physicochem. Eng. Asp. 112(1), 31 (1996)

17. J.H. Jander, P.S. Schmidt, C. Giraudet, P. Wasserscheid, M.H. Rausch, A.P. Fröba, Int. J. Hydrogen Energy 46(37), 19446 (2021)

18. P.K. Bikkina, O. Shoham, R. Uppaluri, J. Chem. Eng. Data 56(10), 3725 (2011)

19. A. Zolghadr, M. Escrochi, S. Ayatollahi, J. Chem. Eng. Data 58(5), 1168 (2013)

20. B. Song, J. Springer, J. Colloid Interface Sci. 184(1), 64 (1996)

21. L.L. Schramm, D.B. Fisher, S. Schurch, A. Cameron, Colloids Surf. A Physicochem. Eng. Asp. 94(2), 145 (1995)

22. A.M. Sayed, K.B. Olesen, A.S. Alkahala, T.I. Sølling, N. Alyafei, J. Petrol. Sci. Eng. 173, 1047 (2019)

23. I. Egry, G. Lohöfer, P. Neuhaus, S. Sauerland, Int. J. Thermophys. 13(1), 65 (1992) 
24. I. Egry, G. Lohoefer, G. Jacobs, Phys. Rev. Lett. 75(22), 4043 (1995)

25. S.K. Chung, D.B. Thiessen, W.K. Rhim, Rev. Sci. Instrum. 67(9), 3175 (1996)

26. I. Egry, Mater. Trans. JIM 45(12), 3235 (2004)

27. A. Goebel, K. Lunkenheimer, Langmuir 13(2), 369 (1997)

28. S. Zeppieri, J. Rodríguez, A.L. López de Ramos, J. Chem. Eng. Data 46(5), 1086 (2001)

29. Y.T.F. Chow, PhD thesis, Imperial College London (2017)

30. B. Edlén, Metrologia 2(2), 71 (1966)

31. M. Daimon, A. Masumura, Appl. Opt. 46(18), 3811 (2007)

32. M.E. Thomas, S.K. Andersson, R.M. Sova, R.I. Joseph, Infrared Phys. Technol. 39(4), 235 (1998)

33. M. Kodera, K. Watanabe, M. Lassiège, S. Alavi, R. Ohmura, J Ind Eng Chem 81, 360 (2020)

34. R. Aveyard, D.A. Haydon, Trans. Faraday Society 61, 2255 (1965)

35. G. Wiegand, E.U. Franck, Ber. Bunsenges. Phys. Chem. 98(6), 809 (1994)

36. F.L. Pedrotti, L.M. Pedrotti, L.S. Pedrotti, Introduction to Optics, 3rd edn. (Cambridge University Press, Cambridge, 2017)

37. R. Belda, J.V. Herraez, O. Diez, Phys. Chem. Liq. 43(1), 91 (2005)

38. S. Kurtz Jr., A. Wikingsson, D. Camin, A. Thompson, J. Chem. Eng. Data 10(4), 330 (1965)

39. R.M. Waxler, C.E. Weir, J Res Natl Bur Stand A Phys Chem 67A(2), 163 (1963)

40. T.M. Aminabhavi, V.B. Patil, M.I. Aralaguppi, H.T.S. Phayde, J. Chem. Eng. Data 41(3), 521 (1996)

41. H.B. Chae, J.W. Schmidt, M.R. Moldover, J. Phys. Chem. 94(25), 8840 (1990)

42. W.M.M. Yunus, Y.W. Fen, L.M. Yee, Am. J. Appl. Sci. 6(2), 328 (2009)

43. H.J. Achtermann, T.K. Bose, H. Rögener, J.M. St-Arnaud, Int. J. Thermophys. 7(3), 709 (1986)

44. A. Michels, J. Hamers, Physica 4(10), 995 (1937)

Publisher's Note Springer Nature remains neutral with regard to jurisdictional claims in published maps and institutional affiliations. 